\title{
GW23-e1651 INTERLEUKIN-6 PROMOTOR-634C/G POLYMORPHISM IS ASSOCIATED WITH ATRIAL FIBRILLATION IN ELDERLY HAN CHINESE PATIENTS WITH ESSENTIAL HYPERTENSION
}

doi:10.1136/heartjnl-2012-302920b.22

${ }^{1}$ Min Pan, ${ }^{1}$ Min-Hui Jiang, ${ }^{2}$ Shu-Ping Gao, ${ }^{1}$ Jian-Hua Zhu. ${ }^{1}$ Affiliated Hospital of Nantong University; ${ }^{2}$ Ningxia People's Hospital

Objectives There is an accumulating body of evidence indicating strong association between inflammation and the pathogenesis of atrial fibrillation (AF). Interleukin-6 (IL-6) is a pleiotropic cytokine, functions as a mediator of inflammatory response and has both pro-inflammatory and anti-inflammatory properties. Three single nucleotide polymorphisms (SNPs) in the IL-6 promoter region $(-597 \mathrm{G} / \mathrm{A}$; $-634 \mathrm{C} / \mathrm{G}$ and $-174 \mathrm{G} / \mathrm{C})$ have been reported to influence IL-6 transcription, and $-174 \mathrm{G} / \mathrm{C}$ was in tight linkage disequilibrium with $-597 \mathrm{G} / \mathrm{A}$. The $-174 \mathrm{C}$ allele is extremely rare and the $-634 \mathrm{C}$ allele is common in eastern Asian populations. The aim of the present study is to investigate the association of $-634 \mathrm{C}>\mathrm{G}$ polymorphism of IL-6 gene with AF in elderly Han Chinese patients with essential hypertension $(\mathrm{EH})$.

Methods A total of 169 elderly patients with EH were eligible for this study. Patients with AF $(n=75)$ were allocated to the AF group, and 94 subjects without AF to the control group. The PCRbased restriction fragment length polymorphism (PCR-RFLP) technique was used to assess the genotypes frequencies.

Results The distribution of the IL- $6-634 \mathrm{C}>\mathrm{G}$ genotypes (CC, CG, and GG) was $67.02 \%, 30.85 \%$, and $2.13 \%$ in the controls, and $50.67 \%, 40.00 \%$, and $9.33 \%$ in AF subjects, respectively $(p=0.0312)$. The frequency of the $G$ allele in the AF group was significantly higher than that in the control group $(29.33 \%$ vs $17.55 \%, p=0.0103)$. Compared with the wild type CC, the G allele carriers (CG+GG genotypes) had increased risk of $\mathrm{AF}$ in both unadjusted ( $\mathrm{OR}=1.98,95 \% \mathrm{CI} 1.06$ to $3.69, \mathrm{p}=0.0312)$ and adjusted analyses $(\mathrm{OR}=1.93,95 \% \mathrm{CI} 1.04$ to $3.57, \mathrm{p}=0.0364)$.

Conclusions These findings suggest that IL-6 -634C $>\mathrm{G}$ polymorphism is associated with $\mathrm{AF}$ and the $\mathrm{G}$ allele is an independent risk for AF in elderly Han Chinese patients with EH. 